\title{
A New Species of Argophyllum (Argophyllaceae) with Notes on the Species from New Caledonia and Nickel Hyperaccumulation
}

\author{
Yohan Pillon $^{1, *}$ and Vanessa Hequet ${ }^{2}$ \\ 1 LSTM, IRD, INRAE, CIRAD, Institut Agro, Univ. Montpellier, 34398 Montpellier, France \\ 2 AMAP, IRD, Herbier NOU, 98848 Nouméa, New Caledonia; vanessa.hequet@ird.fr \\ * Correspondence: yohan.pillon@ird.fr
}

Citation: Pillon, Y.; Hequet, V. A New Species of Argophyllum (Argophyllaceae) with Notes on the Species from New Caledonia and Nickel Hyperaccumulation. Plants 2021, 10, 701. https://doi.org/ 10.3390/plants10040701

Academic Editor:

Celestino Quintela-Sabarís

Received: 25 February 2021

Accepted: 27 March 2021

Published: 5 April 2021

Publisher's Note: MDPI stays neutral with regard to jurisdictional claims in published maps and institutional affiliations.

Copyright: (C) 2021 by the authors. Licensee MDPI, Basel, Switzerland. This article is an open access article distributed under the terms and conditions of the Creative Commons Attribution (CC BY) license (https:// creativecommons.org/licenses/by/ $4.0 /)$.

\begin{abstract}
The taxonomy of Argophyllum (Argophyllaceae) in New Caledonia is reviewed here. All names validly published in Argophyllum in this archipelago are discussed and lectotypified when necessary. A new species is described, Argophyllum riparium (The LSID for the name Argophyllum riparium is: 77216335-1) Pillon and Hequet sp. nov. Argophyllum grunowii and A. ellipticum are both species complexes in which several species previously recognized are included here as well. Seven species are recognized in New Caledonia: A. brevipetalum, A. ellipticum, A. grunowii, A. montanum, A. nitidum, $A$. riparium and $A$. vernicosum, all endemic. Leaf nickel content of $A$. riparium can exceed $1000 \mu \mathrm{g} \cdot \mathrm{g}^{-1}$, which makes this species a nickel hyperaccumulator. Measurements with a handheld X-Ray Fluorescence (XRF) spectrometer confirmed that this was also the case for all other species from New Caledonia, except A. nitidum. An identification key of New Caledonian species is provided.
\end{abstract}

Keywords: island; metal hyperaccumulation; Pacific; serpentine; systematics; ultramafic

\section{Introduction}

Argophyllum J.R.Forst. and G.Forst. [1] (p. 29) is a genus of shrubs and small trees that has traditionally been placed in Saxifragaceae, or sometimes in Escalloniaceae or Grossulariaceae. Molecular phylogenetic studies [2] have indicated affinities with the genus Corokia A. Cunn. [3] (p. 248) and the two genera form the family Argophyllaceae [4] in the order Asterales [5] (APG I and subsequent others). Argophyllaceae are closely related to two other small families [2,6,7]: Phellinaceae, with a single genus of ten species all endemic to New Caledonia [8-10], and Alseuosmiaceae, with four to five genera and ten species from eastern Australia, New Guinea, New Zealand and New Caledonia [11-13]. The exact relationships between Argophyllaceae, Phellinaceae and Alseuosmiaceae, which form the "APA clade" or Alseuosmiineae Shipunov [14] (p. 63), are still not clear [15].

The genus Argophyllum was described by Johann and Georg Forster, two German botanists who travelled with James Cook during his second voyage around the world. They collected material in New Caledonia that they subsequently described as Argophyllum nitidum J. R. Forst. and G. Forst. [1] (p. 29). A second species, A. ellipticum Labill. [16] (p. 39), was collected and described by Labillardière, a member of Bruni d'Entrecasteaux's voyage in search of the La Pérouse expedition. The genus was last revised in its entirety by Zemann [17], who recognized ten species in Australia and New Caledonia. Leaf cuticle attributed to Argophyllum have been described from the Miocene of New Zealand [18]. The species in Australia have recently been revised with a total of eleven endemic species [19,20]. Guillaumin [21] revised the New Caledonia species as part of his revision of New Caledonian "Saxifragaceae" and recognised seven species, to which he subsequently added three new species [22]. Here, we provide a taxonomic update of New Caledonian Argophyllum to complete work on the genera from New Caledonia previously included in "Saxifragaceae" [23,24]. 
High nickel content was previously reported in the leaves of New Caledonian Argophyllum [25,26]. However, because the taxonomy of the genus was not clear at that time, there was uncertainty regarding the number and the names of the species that can actually be considered as nickel hyperaccumulators $[27,28]$. Here, we provide new analyses of herbarium specimens [29] and field-collected material to update the list of nickel hyperaccumulators in the genus Argophyllum.

\section{Materials and Methods}

Measurements, shapes and colours of the different organs are based on the examination of herbarium material and field observations. All herbarium specimens of Argophyllum present at NOU and P were examined (for herbarium acronyms: [30]). Additional type material was viewed online.

Nickel content in herbarium specimens was measured with a handheld X-Ray Fluorescence (XRF) spectrometer $[28,31]$. For each species, we selected specimens to represent their morphological, geographical and ecological range. For a putative new species, one leaf from five individuals was collected in the field, dried, ground to powder, digested in $\mathrm{HNO}_{3} / \mathrm{H}_{2} \mathrm{O}_{2}$ and analysed by Microwave Plasma-Atomic Emission Spectrometer (MPAES) [32].

\section{Results}

Examination of herbarium specimens and observations in the field suggests that seven endemic species should be recognized in the genus. Some material resembling A. vernicosum Däniker [33] (p. 165) but with larger leaves, thicker twigs, sparser branching, sepals with a different shape, and a riparian ecology are placed in a new taxon described below, A. riparium Pillon and Hequet sp. nov.

Measurements with an XRF spectrometer indicate that the nickel content in dried leaves exceeds $1000 \mu \mathrm{g} \cdot \mathrm{g}^{-1}$ in at least some individuals of six species: A. brevipetalum, A. ellipticum, A. grunowii, A. montanum, $A$. riparium sp. nov. and $A$. vernicosum (Table 1 ). The range of values observed between theses six species are similar. The median value for all species is below $1000 \mu \mathrm{g} \cdot \mathrm{g}^{-1}$ and the highest value, $6670 \mu \mathrm{g} \cdot \mathrm{g}^{-1}$, is observed in A. ellipticum. The only exception is A. nitidum, where values were consistently below the level of detection. Values observed with MP-AES on five individuals of Argophyllum riparium sp. nov. confirmed that it is a nickel hyperaccumulator: 1498, 1520, 1678, 2111 and $2214 \mu \mathrm{g} \cdot \mathrm{g}^{-1}$ nickel in dried leaves.

Table 1. Nickel content $\left(\mu \mathrm{g} \cdot \mathrm{g}^{-1}\right)$ measured from herbarium specimens with a handheld X-Ray Fluorescence (XRF) spectrometer. Values are in $\mu \mathrm{g} \cdot \mathrm{g}^{-1}$. LOD: Level of Detection. See Appendix A for individual values.

\begin{tabular}{cccccc}
\hline Species & $\begin{array}{c}\text { Number of } \\
\text { Specimens }\end{array}$ & Min (Ni) & Max (Ni) & Mean (Ni) & Median (Ni) \\
\hline A. brevipetalum & 4 & <LOD & 1675 & 483 & 129 \\
A. ellipticum & 12 & <LOD & 6670 & 1215 & 371 \\
A. grunowii & 40 & <LOD & 5694 & 1027 & 538 \\
A. montanum & 10 & <LOD & 1110 & 316 & 89 \\
A. nitidum & 9 & <LO & $<$ LOD & $<$ LOD & $<$ LOD \\
A. riparium & 4 & 173 & 1667 & 708 & 497 \\
A. vernicosum & 10 & 116 & 1872 & 608 & 387 \\
\hline
\end{tabular}

\section{Discussion}

Seven species of Argophyllum, all endemic, can be recognized in New Caledonia. Two of them are variable and appear as species complexes: A. ellipticum or A. grunowii. It is not clear if this complexity may be caused by, e.g., hybridization $[34,35]$ or cryptic species [36,37]. 
The obscure taxonomy of the genus led to uncertainty and discrepancy regarding the number and identity of the nickel hyperaccumulating species in different publications: A. grunowii and A. laxum [25,26,38], A. ellipticum [28], A. brevipetalum, A. grunowii, A. latifolium, A. laxum, A. montanum and A. vernicosum [27]. Novel measurements associated with a new taxonomy indicate that the nickel content in dried leaves exceeds $1000 \mu \mathrm{g} \cdot \mathrm{g}^{-1} \mathrm{in}$ at least some specimens of six species that can, therefore, be considered as nickel hyperaccumulators [39]: A. brevipetalum, A. ellipticum, A. grunowii, A. montanum, A. riparium sp. nov. and $A$. vernicosum. These six species are ultramafic obligates, except $A$. ellipticum, which can also occur on other substrates. The seventh species of the genus in New Caledonia, A. nitidum, never occurs on ultramafic substrates and consistently has nickel content below the level of detection. The genus Argophyllum is therefore similar to Geissois Labill. [16] (p. 50) (Cunoniaceae): Out of the 13 species endemic to New Caledonia, seven of the eight species occurring on ultramafic substrates are nickel hyperaccumulators [40].

Although six species of Argophyllum qualify as nickel hyperaccumulators, their nickel content do not reach the high values observed in some other New Caledonian species [27]. The genus could nevertheless be used for phytoremediation, phytoextraction or ecological restoration, but its multiplication and cultivation has apparently not yet been tested in New Caledonia [41]. The cultivation of the Australian species is considered easy [42].

\section{Taxonomic Section}

- Argophyllum J. R. Forst. and G. Forst. [1] (p. 29). Type:-Argophyllum nitidum J. R. Forst. \& G. Forst.

non Argophilum Blanco [43] (p. 186) = Aglaia Lour. [44] (p. 173)

- Sectional names

1. Sect. Argophyllum

sect. Brachycalyx Zemann [17] (p. 271) nom. inval. Schltr.

2. Sect. Dolichocalyx Zemann [17] (p. 271). Type [17] (p. 289):-Argophyllum laxum

Zemann [17] did not provide a formal Latin diagnosis for either of the two sections. Section Brachycalyx included species from Australia and New Caledonia: Argophyllum ellipticum, A. lejourdani F.Muell. [45] (p. 33), A. nitidum, A. nullumense Baker [46] (p. 439) and A. cryptophlebum Zemann [17] (p. 283), whereas section Dolichocalyx included only New Caledonian species: A. montanum, A. schlechterianum, A. grunowii, A. laxum and A. latifolium. Because it includes the type of the genus, section Brachycalyx is not a valid name [47] (article 22.2). Zemann distinguished the two sections based on the length of the petals: calyx much shorter than the corolla in section Brachycalyx, calyx half as long but generally equal to the corolla in sect. Dolichocalyx. These sections are not maintained. They may not be reciprocally monophyletic groups, or this would imply that Australian and New Caledonian Argophyllum are not monophyletic groups. Additionally, calyx length is a labile character, i.e., it is variable within A. ellipticum.

- Argophyllum brevipetalum Guillaumin [22] (p. 25). Type:-New Caledonia, cours moyen de la Tontouta, le long des rives, \pm 50 m, 14 December 1940, Virot 375 (holo-: P00537620!)

Argophyllum brevipetalum is very similar to A. ellipticum, with which it shares the combination of yellow petals and hairy reddish coriaceous leaves, with the entire margin and inflorescences covered with red hairs. However, it occurs at a much lower elevation and differs in its smaller proportions (smaller leaves, slender petiole and stem). Guillaumin [22] (p. 25) segregated $A$. brevipetalum from A. ellipticum because of its calyx that is as long as its corolla, but this character is variable within A. ellipticum and is therefore not a reliable character alone on which to separate them.

- Argophyllum ellipticum Labill. [16] (p. 39). Lectotype (designated here):-New Caledonia, Labillardière s.n. (P00537623!, iso-: P00537624!, P00537625!) 
Argophyllum ellipticum var. comptonii Baker f. [48] (p. 299), syn. nov. Type:-New Caledonia, Tonine, 30 September 1914, Compton 1938 (holo-: BM001254561!)

Argophyllum ellipticum var. ovatum Pamp. [49] (p. 81), syn. nov. Type:-New Caledonia, Labillardière s.n. (holo-: FI018226!)

Argophyllum ellipticum var. rigidum Däniker [50] (p. 160), syn. nov. Type:-New Caledonia, auf des sud ost crête des Mt Humboldt, 7 November 1924, Däniker 547 (holoZ000015794!, iso-: Z000015795!)

Argophyllum rufum Vieill. ex Zemann [17] (p. 282) nom. nud.

Argophyllum obovatum Brongn. \& Gris ex Guillaumin [51] (p. 60) [52] (p. 134) nom. nud. Argophyllum vernicosum var. obovatum (Brongn. \& Gris ex Guillaumin) Guillaumin [21] (p. 277) nom. nud.

Argophyllum ellipticum is characterized by the combination of yellow petals, sepals much shorter or as long as petals, hairy coriaceous leaves (generally red and obovate in the most typical forms) with the entire margin and inflorescences generally covered with red hairs. Argophyllum ellipticum is widespread across the main island of New Caledonia, at mid- to high elevation, generally on ultramafic substrates, but occasionally on other, mostly poor, soils. It is a variable taxon, more robust in its dimension than the rare low-elevation A. brevipetalum. Specimens collected in the northeast of the island on non-ultramafic substrates, including the type of the species and of var. comptonii, have particularly short sepals (three times shorter than petals) and inflorescences with a long basal peduncle ramifying very distally. Most material collected on ultramafic substrates from Mont Humboldt and Mont Kouakoué, including the type of $A$. ellipticum var. rigidum, have leaves that are often revolute and whitish underneath, slender sepals (almost as long as petals), and shorter inflorescence ramified nearer the base. In locations in between these, generally on ultramafic substrates, the material displays a range of intermediate characters, which make this taxon difficult to divide further.

The name Argophyllum obovatum was published without a description and the specimen cited by Guillaumin [51] (p. 60), Balansa 1814 (P00537626!, P00537627!) belongs to A. ellipticum. Argophyllum rufum has never been published with a description and was treated by Zemann [17] as a synonym of A. ellipticum.

- Argophyllum grunowii Zahlbr. [53] (p. 278). Type:-New Caledonia Thio, auf serpentinbergen, September 1884, Grunow s.n. (holo-: W1887-0007474!, iso-: NSW923234!)

Argophyllum acinetochromum Guillaumin [22] (p. 24), syn. nov. Type:-New Caledonia, pentes sud du Mont Kaala, \pm 500 m, 2 November 1943, Virot 1320 (holo-: P00537619!; iso-: NOU008828!)

Argophyllum amoenum var. ovatum Vieill. ex Guillaumin [52] (p. 133) nom. nud.

Argophyllum brevistylum Guillaumin [22] (p. 25), syn. nov. Type:-New Caledonia, près du sommet sud du Mont Kaala, \pm 1000 m, 2 November 1943, Virot 1318 (holo-: P00537621!; iso-: A01154254!, P00537622!)

Argophyllum latifolium Vieill. ex Zemann [17] (p. 285), syn. nov. Lectotype (designated here):- New Caledonia, ad montes prope Wagap, 1861-1867, Vieillard 2199 (P00537630!, iso-: P00537631!, P00537632!). Remaining syntypes:-New Caledonia Kanala, Deplanche 61 (P00537634!, P00537635!, P00537636!)

Argophyllum laxum Schltr. [54] (p. 118), syn. nov. Lectotype (designated here):?New Caledonia, Auf den Bergen bei Paita, 400 m, 7 October 1902, Schlechter 14962 (P00537637!, iso:- BR699808!, E00346922!, HBG515583!, K000739390!, W1907-0011817!). Remaining syntypes:-New Caledonia, Auf den Bergen am Ngoye, 150 m, 30 November 1902, Schlechter 15149 (B100715954!, BR699807!, E00346923!, HBG515582!, P00537638!, K000739391!, W10970011659 !)

Argophyllum laxum var. subintegrifolium Baker f. [48] (p. 300), syn. nov. Type:-New Caledonia, riv. Ngoye, 20 October 1914, Compton 2095 (holo-: BM000600291!)

Argophyllum schlechterianum var. vestitum Baker f. [48] (p. 299), syn. nov. Type:-New Caledonia, Taom, 2 December 1914, Compton 2298 (holo-: BM001254563!)

Argophyllum splendens Vieill. ex Guillaumin [52] (p. 134) nomen. nud. 
Argophyllum grunowii is characterized by the combination of yellow petals, and leaves and inflorescences covered with greyish hairs. Plants from southwest New Caledonia (e.g., type of A. laxum) have large leaves and inflorescences much longer than the leaves. Plants from the eastern coast (e.g., type of $A$. latifolium) have large leaves but inflorescences shorter than the leaves. Plants from the northwest (e.g., the types of A. acinetochromum and A. brevistylum) have medium-sized leaves with a very dense indumentum underneath and inflorescences about the length of the leaves. In spite of these apparent geographical trends, there are many intermediate forms and variability found within populations. Although not entirely satisfactory, a broad concept is adopted here. Argophyllum grunowii is widespread across the main island of New Caledonia, at low to high elevation, only on ultramafic substrates.

The name Argophyllym amoenum (published as "amœnum") apparently never appeared in print except in combination with var. ovatum. The name was published without a description and the single specimen cited, Vieillard 2639 (P06309317!, P06309318!, P06309319!, P06309320!, P06309321!, P06309322!) belongs to A. grunowii. The collection number for the type collection of Argophyllum laxum var. subintegrifolium is 2095 on the herbarium label but was printed as 2905 in Rendle et al. [48]. The name $A$. splendens was published without a description and was treated by Guillaumin [52] as a synonym of A. laxum. Zemann [17] cited two collections for A. latifolium, Vieillard 2199 and Deplanche 61; one sheet of Vieillard 2199 is chosen here as a lectotype. Schlechter [54] cited two of his own collections for A. laxum: Schlechter 14962 and 15149. The first one (from Païta) is chosen over the second one (from Ngoye), as it was collected in the area where most of the typical forms have been collected.

- $\quad$ Argophyllum montanum Schlechter [54] (p. 118). Lectotype (designated here):-New Caledonia, Auf den Bergen bei Yaouhé, 700 m, 15 October 1902, Schlechter 15032 (B100715955!; iso-:P00537639!, K000739388!)

Argophyllum schlechterianum Bonati \& Petitmangin [55] (p. 650). Type:-New Caledonia, forêt du mont Dzumacs, 900 m, October 1906, Franc 566 (holo-: Z00054678!)

Argophyllum montanum is characterized by the combination of yellow petals, glabrous leaves and inflorescences covered by greyish hairs. Argophyllum montanum occurs in the southern part of New Caledonia, at low to mid elevation, only on ultramafic substrates.

The type specimen is unusual in having some toothed leaves. We follow Guillaumin [21] in treating $A$. schlechterianum as a synonym of $A$. montanum.

- Argophyllum nitidum J.R.Forst. and G.Forst. [1] (p. 30). Argophyllum nitidum var. nitidum J.R.Forst. \& G.Forst. Lectotype (designated here): New Caledonia, Forster s.n. (BM000600294!, iso-: BM000600292!, BM00600293!, K000739389! B100296104!, BW04962010!, W0017375!). See Nicolson and Fosberg [56] for a complete list of putative isolectotypes.

Argophyllum sericeum Poir. [57] (p. 449) nomen. nud.

This species here is considered as endemic to New Caledonia, although some of the Australian species have sometimes been treated as varieties of $A$. nitidum. It is the only species from New Caledonia with white petals. Its calyx is particularly short $(<2 \mathrm{~mm})$. Its leaves are more chartaceous with a finer silvery indumentum than other species. Its inflorescences are silvery too, sometimes tinted with red. Argophyllum nitidum is widespread across the main island of New Caledonia, from Mount Koghis to Balade, and it is most common in the northern part, on non-ultramafic substrates and at low- to mid-elevation.

- Argophyllum vernicosum Däniker [33] (p. 165). Type:-New Caledonia, im dichten hohen Gebüsch der Crêten in 600 m Meereshöhe am Mt Koghi, 1 February 1926, Däniker 2725 (holo-: Z000015797!, iso-: Z000015798!, Z000015799!)

Argophyllum ellipticum var. oblongifolium Brongn. and Gris ex Guillaumin [52] (p. 133). nom. nud.

Argophyllum vernicosum is characterized by the combination of yellow petals, short calyx, glabrous shiny leaves and inflorescences covered with reddish hairs. Argophyllum 
vernicosum occurs in the southern part of New Caledonia, at mid- to high elevation, on ultramafic substrates.

The name Argophyllum ellipticum var. oblongifolium (originally "oblongifolia") was published without a description; the single specimen cited, Balansa 1816 (P0537640!), belongs to A. vernicosum, and we therefore follow Guillaumin [21] in considering A. ellipticum var. oblongifolium as a synonym of $A$. vernicosum.

- $\quad$ Argophyllum riparium Pillon and Hequet, sp. nov (Figure 1)

Diagnosis: A species most similar to Argophyllum vernicosum Däniker, from which it differs by its larger leaves (generally $\geq 10 \mathrm{~cm}$ long), thicker twigs (at least 3-4 $\mathrm{mm}$ in diameter) and sparse branching. Its sepals are narrowly attenuated, nearly as long as petals, rather than shortly acute and not more than half the size of petals as in A. vernicosum.

Type: New Caledonia, Forêt de la rivière bleue près d'un creek, 23 April 1970, Veillon 2137 (holo-: P04445141!, iso-: NOU024016 !).

Description: Shrub 1-2 m tall. Stipules absent. Hairs on new growth brown or rusty. Branchlet terete, thick, 3-4 mm in diameter at the insertion of inflorescences, reddish-brown. Leaves alternate, discolorous, at the end of the twigs. Petiole $14-41 \mathrm{~mm} \times 2-2.5 \mathrm{~mm}$. Leaf blade elliptic 9-18 cm $\times 2.5-6.5 \mathrm{~cm}$, base acute, apex acute or retuse, 5-8 secondary veins on either side of midrib, margin entire to slight wavy, sometimes with a few teeth, few hairs invisible to the naked eyes (binocular $\times 4$ ), glabrous and shiny on both sides. Inflorescence axillary, paniculate, densely reddish tomentose, 8-14 cm long, 15-60 flowers, primary axis $1 / 2-2 / 3$ of the length of the inflorescence, secondary axis $10-50 \mathrm{~mm}$ long, branching spreading at $30-60^{\circ}$ from adjacent branch. Bracts subtending primary ramifications of the inflorescence $4 \times 0.5 \mathrm{~mm}$, persistent. Floral bracts $2-3 \mathrm{~mm},(? 1-) 2$ per flower. Flower apparently bisexual, actinomorphic. Pedicels $2 \mathrm{~mm}$ long, flowering hypanthium cupular, $2.5 \mathrm{~mm}$ diameter. Calyx lobes $2.5 \mathrm{~mm}$ long; petals $3 \mathrm{~mm}$ long, yellow, corolla appendage yellow, $1 \mathrm{~mm}$ long. Stamen antesepalous, staminal filament $0.8 \mathrm{~mm}$ long, anthers $0.4 \mathrm{~mm}$ long. Style simple, $1.2 \mathrm{~mm}$ long, stigma swollen, entire, globose. Seed unknown.

Ecology and conservation: This species has been found in two locations (Figure 2). Most collections came from within the Rivière Bleue Provincial Park, where it grows near the rainforest edge on the banks of "rivière bleue". All these collections may have been made from the same population. It appears in small, scattered groups of individuals, and its population size may be small. It was found recently in a second location in Plaine des lacs, growing along a stream close to the Kuebini river, $35 \mathrm{~km}$ apart from the type locality. Its ecosystem is undisturbed, and one of the two sites occurs within a protected area where the most likely disturbance might be floods, which can be severe during tropical storms. Its conservation status will be evaluated by the Plant Red List Authority-New Caledonia, and it could qualify as Vulnerable (VU D2).

Paratypes: New Caledonia, haute rivière bleue, 200m, 6 August 1951, Baumann 15031 (P03609388!); ibid., 5 August 1951, Baumann 15034 (P03609386!); Rivière Bleue S $22^{\circ} 5^{\prime}-22^{\circ} 7^{\prime}$, E 166 37'-166 44', 150 m, 1965, Bernardi 9317 (P06234104!); Parc Provincial de la Rivière Bleue, S 22 ${ }^{\circ} 5^{\prime} 47.4^{\prime \prime}$, E 166 38'16.4", 200 m, 8 November 2018, Pillon $\mathcal{E}$ Isnard 1480 (P, NOU); Plaine des lacs: Kuebini S 22 ${ }^{\circ} 13^{\prime} 9.89^{\prime \prime}$, E 166 57'15.24", 350 m, 23 September 2020, Hequet 4668 (NOU107206!); Parc de la rivière bleue, S 22 05'49”, E 166 38'16", 200 m, 18 March 2020, Hequet 4662 (NOU106963 !). 


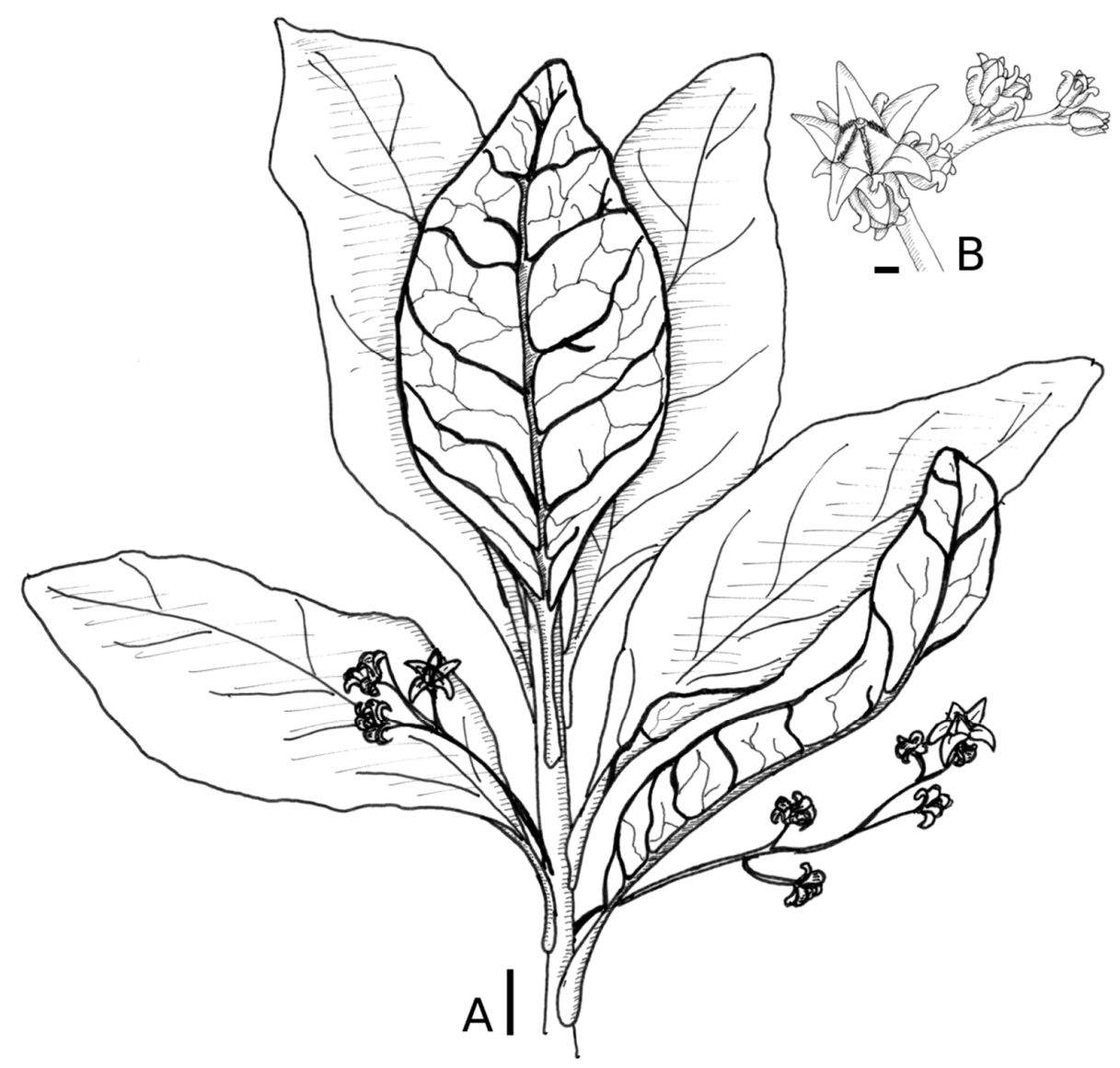

Figure 1. Argophyllum riparium Pillon and Hequet. (A) flowering branch, scale $=2 \mathrm{~cm}$; (B) portion of an inflorescence, scale $=1 \mathrm{~mm}$. Drawn from Hequet 4662 by Thierry Sanchiz.

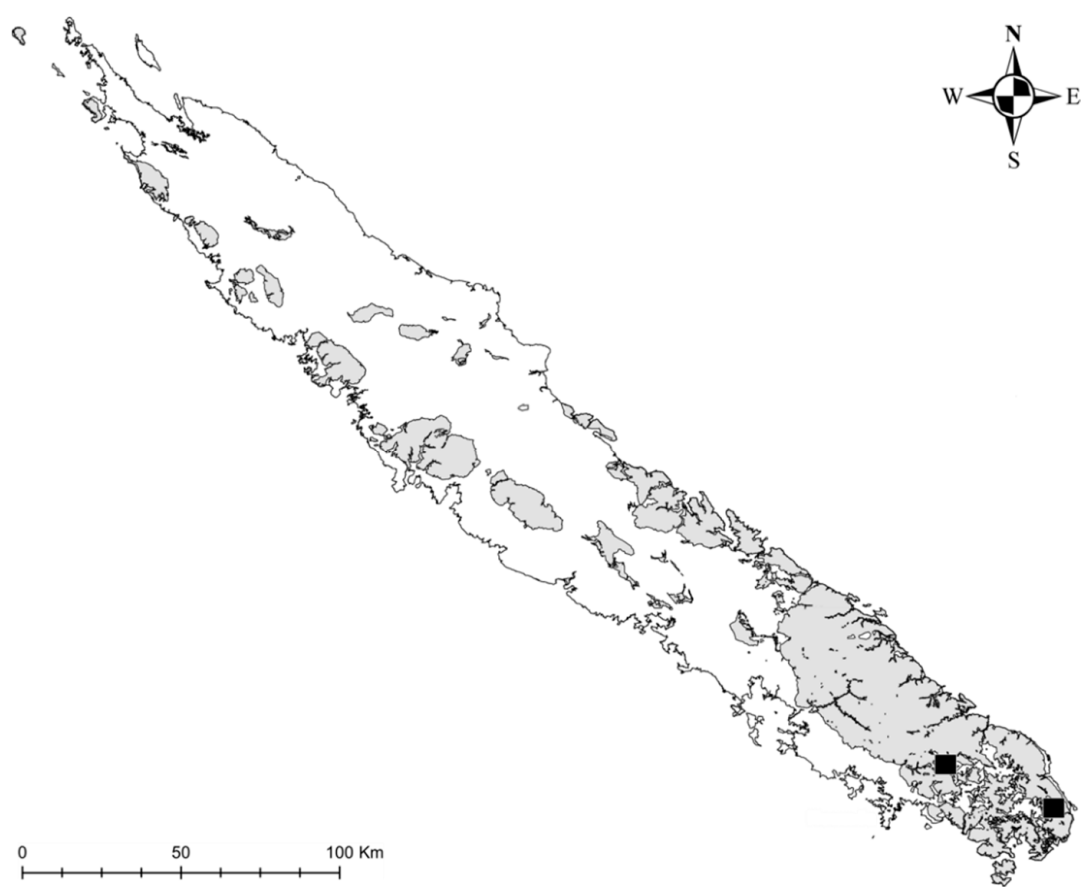

Figure 2. Distribution of Argophyllum riparium Pillon and Hequet (squares). Grey areas indicate ultramafic rocks. 


\section{Identification Key to the Species of Argophyllum in New Caledonia}

1. Leaf chartaceous, apex pointed, only primary and secondary veins distinct, leaf and inflorescences silky silvery (rarely tinted in red), petals white, calyx short $(<2 \mathrm{~mm})$, non-ultramafic substrates $\mathbf{1}^{\prime}$. Leaf coriaceous, apex pointed or rounded, venation network distinct, leaves and inflorescences glabrous or covered with silver or red hairs, petals yellow, calyx short or as long as petals, generally on ultramafic substrate (occasionally on non-ultramafic but then on poor substrate for A. ellipticum) 2. Inflorescences covered with red hairs, leaf margin generally entire

$\mathbf{2}^{\prime}$. Inflorescences covered with white hairs, leaf margin entire to markedly toothed

3. Leaf blade covered with red hairs underneath, occasionally white when mature

$3^{\prime}$. Leaf blade glabrous, shiny underneath

4. Overall plant slender, twig slender (diameter $2.5-4 \mathrm{~mm}$ ), leaf $4-10 \mathrm{~cm}$ long, petiole $1.5 \mathrm{~mm}$ in diameter, petals and sepals about the same length, low elevation in Tontouta valley $(<300 \mathrm{~m})$

4'. Plant robust, twigs thicker (diameter 4-6 mm), leaf 5-15 cm long, petiole 2 $\mathrm{mm}$ in diameter, sepals much shorter or as long as petals, widespread, midto high elevation (400-) 800-1600 m

5. Leaves small (mostly $<10 \mathrm{~cm}$ long), twig thin (c. $2 \mathrm{~mm}$ in diameter), sepals shortly acute, never more than half the size of petals, well-ramified shrub $5^{\prime}$. Leaves large (mostly $>10 \mathrm{~cm}$ long), twig thicker (3-4 mm in diameter), sepals narrowly attenuate, nearly as long as petals, sparsely branched shrub 6. Leaf glabrous, leaf margin entire, mostly south of Dzumacs-Yaté $\mathbf{6}^{\prime}$. Leaf blade covered with pale to red hairs, leaf margin entire to toothed, widespread and variable

\section{A. nitidum}

2

3

6

4

5

A. brevipetalum

A. ellipticum

A. vernicosum

A. riparium

A. montanum

A. grunowii

Author Contributions: Conceptualization, Y.P.; validation, Y.P. and V.H.; writing-original draft preparation, Y.P.; writing-review and editing, Y.P. and V.H. All authors have read and agreed to the published version of the manuscript.

Funding: This work was partly funded by the X-TreM project of the CNRS MITI X-Life program (PI: Sylvain Merlot).

Acknowledgments: We thank the curators of herbarium NOU and P for providing access to their collections; Chiara Nepi (herbarium FI), Robert Vogt (herbarium B), Martin Callmander (G), Ranee Prakash (BM) for sending digital images of specimens; Cécile Nowak for help with bibliographical searches; and Thierry Sanchiz for the line drawing. Nickel measurements were performed with the help of Sylvain Merlot, Pierre Jouannais and Anaïs da Costa. Two anonymous reviewers provided insightful comments on an earlier version of the manuscript.

Conflicts of Interest: The authors declare no conflict of interest.

\section{Appendix A}

List of herbarium specimens analysed with an XRF spectrometer with herbarium barcodes and Ni content. Specimens with value above $1000 \mu \mathrm{g} \cdot \mathrm{g}^{-1}$ are underlined.

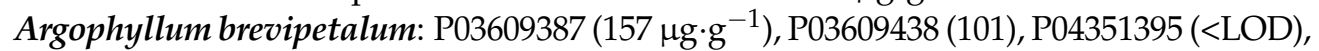
P06234113 (1676); A. ellipticum: P00354794 (309), P03609379 (<LOD), P03609382 (2253), P03609437 (967), P03609439 (3223), P04284932 (608), P04445120 (118), $\overline{\mathrm{P} 04445184(6670)}$, P04445187 (<LOD), P04445188 (<LOD), P06234110 (433), P06234114 (<LOD); A. grunowii: P00217129 (167), P00217183 (1534), P00354673 (1351), P00537619 (207), P00537622 (3879), P00537637 (246), P00537638 (960), P03609286 (41), P03609287 (1581), P03609305 (737), P03609307 (485), P03609314 (320), P03609351 (437), P03609383 (2649), P03609384 (1238), P03609385 (5694), P04284517 (373), P04284521 (796), P04284933 (347), P04284938 (<LOD), P04284939 (489), P04351389 (587), P04351394 (1280), P04351397 (2514), P04351401 (752), P04351404 (782), P04445122 (127), P04445128 (461), P04445152 (229), P04445157 (73), P04445160 (<LOD), P04445164 (896), P04445165 (221), P04445166 (2778), P04445172 (672), P04445175 (1775), P04445179 (213), P04445180 (178), P06234106 (133), P06234119 (3896); A. montanum: 
P03609289 (1110), P03609293 (1076), P03609294 (34), P04284918 (103), P04284920 (<LOD), P04351398 (511), P04445124 (33), P04445145 (50), P04445158 (165), P05563751 (75); A. nitidum: P04284934 (<LOD), P04284935 (<LOD), P04351380 (<LOD), P04445131 (<LOD), P04445133 (<LOD), P04445173 (<LOD), P05563784 (<LOD), P05563794 (<LOD), P06234107 (<LOD); A. riparium: P03609386 (1667), P03609388 (591), P04445141 (402), P06234104 (173); A. vernicosum: P04284916 (132), P04284936 (402), P04351382 (345), P04445117 (1023), P04445162 (116), P05563809 (1872), P05563811 (217), P05563824 (387), P05563829 (977).

\section{References}

1. Forster, J.R.; Forster, G. Characteris Generum Plantarum; White, B., Cadell, T., Elmsly, P., Eds.; Londini: London, UK, 1776.

2. Kårehed, J.; Lundberg, J.; Bremer, B.; Bremer, K. Evolution of the Australasian Families Alseuosmiaceae, Argophyllaceae, and Phellinaceae. Syst. Bot. 1999, 24, 660-682. [CrossRef]

3. Cunningham, A. XXXI.-Florae Insularum Novae Zelandiae Precursor; or a Specimen of the Botany of the Islands of New Zealand. Ann. Nat. Hist. 1839, 3, 244-250. [CrossRef]

4. Kårehed, J. Argophyllaceae. In The Families and Genera of Vascular Plants; Kadereit, J.W., Jeffrey, C., Eds.; Springer: Berlin/Heidelberg, Germany, 2007; Volume 8, pp. 13-18.

5. Angiosperm Phylogeny Group An Ordinal Classification for the Families of Flowering Plants. Ann. Mo. Bot. Gard. 1998, 85, 531-553. [CrossRef]

6. Bremer, B.; Bremer, K.; Heidari, N.; Erixon, P.; Olmstead, R.G.; Anderberg, A.A.; Källersjö, M.; Barkhordarian, E. Phylogenetics of Asterids Based on 3 Coding and 3 Non-Coding Chloroplast DNA Markers and the Utility of Non-Coding DNA at Higher Taxonomic Levels. Mol. Phylogenet. Evol. 2002, 24, 274-301. [CrossRef]

7. Tank, D.C.; Donoghue, M.J. Phylogeny and Phylogenetic Nomenclature of the Campanulidae Based on an Expanded Sample of Genes and Taxa. Syst. Bot. 2010, 35, 425-441. [CrossRef]

8. Barriera, G. Novitates Neocaledonicae. VIII. Taxonomie et Nomenclature du Genre Phelline (Phellinaceae) avec la Description de la Nouvelle Espèce Phelline Barrierei. Candollea 2017, 72, 361-370. [CrossRef]

9. Barriera, G. Phellinaceae. In Flore de la Nouvelle-Calédonie; Faune et Flore Tropicales; Publications scientifiques du Muséum; IRD: Paris, France; Marseille, France, 2020; Volume 27, pp. 234-295.

10. Barriera, G.; Savolainen, V.; Spichiger, R. Phellinaceae. In The Families and Genera of Vascular Plants; Kadereit, J.W., Jeffrey, C., Eds.; Springer: Berlin/Heidelberg, Germany, 2007; Volume 8, pp. 608-610.

11. Kårehed, J. Alseuosmiaceae. In The Families and Genera of Vascular Plants; Kadereit, J.W., Jeffrey, C., Eds.; Springer: Berlin/Heidelberg, Germany, 2007; Volume 8, pp. 7-12.

12. Van Steenis, C.G.G.J. A Synopsis of Alseuosmiaceae in New Zealand, New Caledonia, Australia, and New Guinea. Blumea 1984, 29, 387-394.

13. Shepherd, L.D.; de Lange, P.J.; Townsend, A.; Perrie, L.R. A Biological and Ecological Review of the Endemic New Zealand Genus Alseuosmia (Toropapa; Alseuosmiaceae). N. Z. J. Bot. 2020, 58, 2-18. [CrossRef]

14. Shipunov, A.; Reveal, J.L. Validation of Subordinal Names for "Systema Angiospermarum". Phytotaxa 2011, 16, 63-64. [CrossRef]

15. Soltis, D.E.; Smith, S.A.; Cellinese, N.; Wurdack, K.J.; Tank, D.C.; Brockington, S.F.; Refulio-Rodriguez, N.W.J.B.; Moore, M.J.; Carlsward, B.S.; Bell, C.D.; et al. Angiosperm Phylogeny: 17 Genes, 640 Taxa. Am. J. Bot. 2011, 98, 704-730. [CrossRef]

16. Labillardière, J.-J.H. Sertum Austrocaledonicum; Dominae Huzard: Paris, France, 1824.

17. Zemann, M. Studien Zu Einer Monographie Der Gattung Argophyllum Forst. Ann. Des K.K. Nat. Hofmuseums 1907, $22,270-292$.

18. Pole, M.S. Dispersed Leaf Cuticle from the Early Miocene of Southern New Zealand. Palaeontol. Electron. 2008, 11, 15A.

19. Bean, A.R.; Forster, P.I. A Taxonomic Revision of Argophyllum J.R.Forst. \& G.Forst. (Argophyllaceae) in Australia. Austrobaileya 2018, 10, 207-235.

20. Forster, P.I. Argophyllum Verae (Saxifragaceae), a New Species from Northern Queensland. Austrobaileya 1990, 3, $173-176$.

21. Guillaumin, A. Matériaux pour la Flore de La Nouvelle-Calédonie. LV.-Révision des Saxifragacées. Bull. De La Société Bot. De Fr. 1939, 86, 275-278. [CrossRef]

22. Guillaumin, A.; Virot, R. Contributions à la Flore de la Nouvelle-Calédonie CII.—Plantes Récoltées par M. R. Virot. Mémoires Du Muséum Natl. D'histoire Nat. Ser. Bbotanique 1953, 4, 1-82.

23. Pillon, Y. Nomenclature and Typification in Polyosma (Escalloniaceae) from New Caledonia, with the Description of a New Species. Phytotaxa 2018, 371, 267-272. [CrossRef]

24. Pillon, Y.; Hequet, V. Two New Species of Quintinia (Paracryphiaceae) with Notes on the Species from New Caledonia and Vanuatu. Phytotaxa 2019, 397, 45-54. [CrossRef]

25. Jaffré, T. Composition Chimique Élémentaire des Tissus Foliaires des Espèces Végétales Colonisatrices des Anciennes Mines de Nickel en Nouvelle-Calédonie. Cah. Orstomsérie Biol. 1977, 12, 323-330.

26. Jaffré, T. Etude Écologique du Peuplement Végétal des Sols Dérivés de Roches Ultrabasiques en Nouvelle-Calédonie; Travaux et Documents de l'ORSTOM; ORSTOM: Paris, France, 1980.

27. Gei, V.; Isnard, S.; Erskine, P.D.; Echevarria, G.; Fogliani, B.; Jaffré, T.; van der Ent, A. A Systematic Assessment of the Occurrence of Trace Element Hyperaccumulation in the Flora of New Caledonia. Bot. J. Linn. Soc. 2020, 194, 1-22. [CrossRef] 
28. Jaffré, T.; Pillon, Y.; Thomine, S.; Merlot, S. The Metal Hyperaccumulators from New Caledonia Can Broaden Our Understanding of Nickel Accumulation in Plants. Front. Plant Sci. 2013, 4, 279. [CrossRef]

29. Van der Ent, A.; Echevarria, G.; Pollard, A.J.; Erskine, P.D. X-Ray Fluorescence Ionomics of Herbarium Collections. Sci. Rep. 2019, 9, 4746. [CrossRef]

30. Thiers, B. Index Herbariorum: A Global Directory of Public Herbaria and Associated Staff; New York Botanical Garden: New York, NY, USA, 2020.

31. Gei, V.; Erskine, P.D.; Harris, H.H.; Echevarria, G.; Mesjasz-Przybyłowicz, J.; Barnabas, A.D.; Przybyłowicz, W.J.; Kopittke, P.M.; van der Ent, A. Tools for the discovery of hyperaccumulator plant species and understanding their ecophysiology. In Agromining: Farming for Metals; Van der Ent, A., et al., Eds.; Springer International Publishing: Cham, Switzerland, 2018; pp. 117-133, ISBN 978-3-319-61898-2.

32. García de la Torre, V.S.; Majorel-Loulergue, C.; Rigaill, G.J.; Alfonso-González, D.; Soubigou-Taconnat, L.; Pillon, Y.; Barreau, L.; Thomine, S.; Fogliani, B.; Burtet-Sarramegna, V.; et al. Wide Cross-species RNA-Seq Comparison Reveals Convergent Molecular Mechanisms Involved in Nickel Hyperaccumulation across Dicotyledons. New Phytol. 2021, 229, 994-1006. [CrossRef] [PubMed]

33. Däniker, A.U. Ergebnisse Der Reise von Dr. A.U. U. Däniker Nach Neu-Caledonien Und Den Loyalitäts-Inseln (1924/25). 2. Neue Phanerogamen von Neu-Caledonien Und Den Loyalitäts-Inseln. Vierteljahrsschr. Der Nat. Ges. Zürich 1931, 76, 160-170.

34. Pillon, Y.; Hopkins, H.C.F.; Bradford, J.C. Two New Species of Cunonia (Cunoniaceae) from New Caledonia. Kew Bull. 2008, 63, 419-431. [CrossRef]

35. Pillon, Y.; Munzinger, J.; Amir, H.; Hopkins, H.C.F.; Chase, M.W. Reticulate Evolution on a Mosaic of Soils: Diversification of the New Caledonian Endemic Genus Codia (Cunoniaceae). Mol. Ecol. 2009, 18, 2263-2275. [CrossRef] [PubMed]

36. Pillon, Y.; Hopkins, H.C.F.; Munzinger, J.; Amir, H.; Chase, M.W. Cryptic Species, Gene Recombination and Hybridization in the Genus Spiraeanthemum (Cunoniaceae) from New Caledonia. Bot. J. Linn. Soc. 2009, 161, 137-152. [CrossRef]

37. Swenson, U.; Munzinger, J.; Lowry, P.P.; Cronholm, B.; Nylinder, S. Island Life-Classification, Speciation and Cryptic Species of Pycnandra (Sapotaceae) in New Caledonia. Bot. J. Linn. Soc. 2015, 179, 57-77. [CrossRef]

38. Brooks, R.R. Geobotany and hyperaccumulators. In Plants That Hyperaccumulate Heavy Metals. Their Role in Phytoremediation, Microbiology, Archaeology, Mineral Exploitation and Phytomining; Brooks, R.R., Ed.; CAB International: Wallingford, UK, 1998; pp. 55-94.

39. Van der Ent, A.; Baker, A.J.M.; Reeves, R.D.; Pollard, A.J.; Schat, H. Hyperaccumulators of Metal and Metalloid Trace Elements: Facts and Fiction. Plant Soil 2013, 362, 319-334. [CrossRef]

40. Pillon, Y.; Hopkins, H.C.F.; Rigault, F.; Jaffré, T.; Stacy, E.A. Cryptic Adaptive Radiation in Tropical Forest Trees in New Caledonia. New Phytol. 2014, 202, 521-530. [CrossRef] [PubMed]

41. Gâteblé, G. Flore Ornementale de Nouvelle-Calédonie; Au vent des îles: Tahiti, French Polynesia, 2016; ISBN 978-2-36734-078-4.

42. Kupsch, K. The Silver Leaves of the Rainforest Understorey. Assoc. Soc. Grow. Aust. Plants. Rainfor. Study Group Newsl. 2005, 61, 6.

43. Blanco, M. Flora de Filipinas; Sto. Thomas, por Candido Lopez: Manilla, Philippines, 1837.

44. De Loureiro, J. Flora Cochinchinensis; Ulyssipone: Lisbon, Portugal, 1790.

45. Von Mueller, F. Fragmenta Phytographiae Australiae; Melbourne Auctoritate: Melbourne, Australia, 1863 ; Volume 4.

46. Baker, R.T. Contributions to a Knowledge of the Flora of Australia. No II. Proc. Linn. Soc. N. S. W. 1899, 24, 437-447. [CrossRef]

47. International Code of Nomenclature for Algae, Fungi, and Plants; Turland, N.; Wiersema, J.; Barrie, F.; Greuter, W.; Hawksworth, D.; Herendeen, P.; Knapp, S.; Kusber, W.-H.; Li, D.-Z.; Marhold, K.; et al., Eds.; Regnum Vegetabile; Koeltz Botanical Books: Oberreifenberg, Germany, 2018; Volume 159, ISBN 978-3-946583-16-5.

48. Rendle, A.B.; Baker, E.G.; Moore, S.L.M. A Systematic Account of the Plants Collected in New Caledonia and the Isle of Pines by Prof. R. H. Compton, M.A., in 1914.-Part I. Flowering Plants (Angiosperms). J. Linn. Soc. Lond. Bot. 1921, 45, 245-417. [CrossRef]

49. Pampanini, R. Sassifragacee Dell’Erbario Webb. Nuovo G. Bot. Ital. 1904, 11, 79-82.

50. Däniker, A.U. Ergebnisse Der Reise von Dr. A. U. Däniker Nach Neu-Caledonien Und Den Loyalty-Inseln (1924/6). 4. Katalog Der Pteridophyta Und Embryophyta Siphonogama. II. Vierteljahr. Der Nat. Ges. Zürich 1932, 19, 116-235.

51. Guillaumin, A. Contribution à la Flore de Bourail (Nouvelle-Calédonie). Ann. Du Musée Colonial De Marseille 1911, 9, 55-75.

52. Guillaumin, A. Catalogue des Plantes Phanérogames de la Nouvelle-Calédonie et Dépendances (Île Des Pins et Loyalty). Ann. Du Musée Colonial De Marseille 1911, 9, 77-290.

53. Zahlbruckner, A. Beitrag Zur Flora von Neu-Caledonien, Enthaltend Die von A. Grunow Im Jahre 1884 Daselbst Gesammelten Pflanzen. Ann. Des. K.K. Nat. Hofmuseums 1888, 3, 271-292.

54. Schlechter, R. Beiträge Zur Kenntnis der Flora von Neu-Kaledonien. Bot. Jahrbücher Für Syst. Pflanz. Und Pflanz. 1906, $39,1-274$.

55. Bonati, G.; Petitmengin, M. Sur Quelques Plantes de la Nouvelle-Calédonie. Bull. De L'herbier Boissier 1907, 7, 647-652.

56. Nicolson, D.H.; Fosberg, F.R. The Forsters and the Botany of the Second Cook Expedition (1772-1775); Regnum vegetabile; Gantner: Ruggell, Liechtenstein, 2003; ISBN 978-3-906166-02-5.

57. Poiret, J.L.M. Encyclopédie Méthodique. Botanique. Supplément, Tome I.; H. Agasse: Paris, France, 1810. 